\title{
Tissue engineering of heart valves: PEGylation of decellularized porcine aortic valve as a scaffold for in vitro recellularization
}

\author{
Jianliang Zhou ${ }^{1 * \dagger}$, Shidong $\mathrm{Hu}^{2 \dagger}$, Jingli Ding ${ }^{1}$, Jianjun $\mathrm{Xu}^{1}$, Jiawei Shi ${ }^{3}$ and Nianguo Dong ${ }^{3^{*}}$
}

\author{
* Correspondence: \\ zhoujianliang2010@163.com; \\ dongnianguo2013@163.com \\ †Equal contributors \\ ${ }^{1}$ Department of Cardiothoracic \\ Surgery, the Second Affiliated \\ Hospital of Nanchang University, \\ Nanchang 330006, China \\ ${ }^{3}$ Department of Cardiovascular \\ Surgery, the Union Hospital \\ Affiliated to Tongji Medical College \\ of Huazhong University of Science \\ and Technology, Wuhan 430022, \\ China \\ Full list of author information is \\ available at the end of the article
}

\begin{abstract}
Background: Poly (ethylene glycol) (PEG) has attracted broad interest for tissue engineering applications. The aim of this study was to synthesize 4-arm -PEG-20kDa with the terminal group of diacrylate (4-arm-PEG-DA) and evaluate its dual functionality for decellularized porcine aortic valve (DAV) based on its mechanical and biological properties.
\end{abstract}

Methods: 4-arm-PEG-DA was synthesized by graft copolymerization of linear PEG 20,000 monomers, and characterized by IR ${ }^{1} H$ NMR and ${ }^{13} \mathrm{C}$ NMR; PEGylation of DAV was achieved by the Michael addition reaction between propylene acyl and thiol, its effect was tested by uniaxial planar tensile testing, hematoxylin and eosin (HE) and scanning electron microscopy (SEM). Gly-Arg-Gly-Asp-Ser-Pro-Cys (GRGDSPC) peptides and vascular endothelial growth factor-165 (VEGF 165$)$ were conjugated onto DAV by branched PEG-DA (GRGDSPC-PEG-DAV-PEG-VEGF 165 ).

Results: Mechanical testing confirmed that PEG-cross-linking significantly enhanced the tensile strength of DAV. Immunofluoresce confirmed the GRGDSPC peptides and VEGF 165 were conjugated effectively onto DAV; the quantification of conjunction was completed roughly using spectrophotometry and ELISA. The human umbilical vein endothelial cells (HUVECS) grew and spread well on the GRGDSPC-PEG-DAV-PEG-VEGF 165 .

Conclusions: Therefore, PEGylation of DAV not only can improve the tensile strength of DAV, and can also mediate the conjugation of bioactive molecule (VEGF 165 and GRGDSPC peptides) on DAV, which might be suitable for further development of tissue engineered heart valve.

Keywords: Decellularized valves, Polyethylene glycol, GRGDSPC peptides, VEGF165, Michael addition reaction

\section{Introduction}

Heart valve disease is considered to be among the many factors that are significant for mortality throughout the world. Prosthetic valve implantation utilizing a mechanical prosthetic valve or bioprosthetic heart valve is the most common treatment for valvular heart disease. However, both these approaches have shortcomings. These shortcomings including the risk of: embolism, wear and an inability to grow, which makes them unsuitable for treating congenital heart defects. Consequently, many researchers are now exploring tissue engineering strategies to develop heart valves equivalent $[1,2]$.

(c) 2013 Zhou et al.; licensee BioMed Central Ltd. This is an Open Access article distributed under the terms of the Creative Commons Attribution License (http://creativecommons.org/licenses/by/2.0), which permits unrestricted use, distribution, and reproduction in any medium, provided the original work is properly cited. The Creative Commons Public Domain Dedication waiver (http:// creativecommons.org/publicdomain/zero/1.0/) applies to the data made available in this article, unless otherwise stated. 
Scaffold material is a key factor in preparing heart valves through tissue engineering.

Two categories of materials are used as the scaffold to construct TEHV, namely polymer scaffolds and decellularized allogenic or xenogenic scaffolds [3]. Polymer scaffolds can be derived from biological or synthetic source. Biological polymers possess intrinsic cell compatibility as most are structural components familiar to cells, but also tend to be mechanically weak and difficult to manipulate. Synthetic sources are easily tailored and can be reproduced readily, allowing for novel structural designs that can control mechanical properties, surface topography, and porosity with limitations including uncertainties regarding the degradation rate and products as well as cell compatibility $[4,5]$. Decellularized allogenic and xenogenic scaffolds have natural three-dimensional (3-D) structures and extracellular matrix (ECM) composition. However, because the application of human decellularized allografts is limited due to worldwide organ scarcity, decellularized xenogeneic tissues become highly attractive scaffold materials for tissue engineering (TE) [6]. Among heterogeneous decellularized valves, especially decellularized porcine aortic valves have been used for the preparation of tissue engineered heart valves (TEHV) [7-9], due to their similarity with human heart valves in 3-D structures, ECM composition, and rich source $[6,10,11]$. However, studies found that decellularization may lead to part of the collagen fibers may breaking and a reduction of collagen cross-linking, which in turn may decrease the mechanical strength including ultimate tensile strengths and elastic modulus of the porcine aortic heart valve; in addition loss of extracellular matrix components may affect the adhesion and proliferation of seeded cells on the valve [12-16]. What's more, adequate mechanical stimuli, which can be provided by the dynamic bioreactor in vitro, are conducive to improve the relevant mechanical properties of the valvular complex, such as the mechanical strength and stiffness [17]. Therefore, to overcome these limitations produced by decellularization, it is necessary to find a method or material with good mechanical properties and good biological activity.

Covalent attachment of synthetic polymers to biological macromolecules offers an effective means to modify their properties, providing enhanced functionality for drug delivery and tissue engineering. One of the common approaches to achieve this goal is the covalent attachment of poly (ethylene glycol) (PEG) to therapeutic proteins, termed PEGylation. Therefore, the objective of the present study was to improve the mechanical properties of decellularized porcine aortic valve (DAV) and to promote its endothelialization, which would further facilitate the development of TEHV. To achieve this objective, we aimed to synthesize branched 4-arm poly (ethylene glycol) with the terminal group of diacrylate (PEG-DA), DAV cross-linked by Michael addition reaction between acryloyl with thiol. We also aimed to covalently link the biological signaling molecules (RGD peptide and VEGF) to the decellularized porcine aortic valve.

\section{Materials and methods}

\section{Materials}

PEG 20000, tert-octylphenylpolyoxyethylen (Triton-100), ethylenediaminetetraacetic acid (EDTA), RNase A, DNase, and human recombinant VEGF $_{165}$ were purchased from Sigma. N-succinimidyl-S-acetylthioacetate (SATA) was purchased from Pierce (Rockford, IL, USA). Gly-Arg-Gly-Asp-Ser-Pro-Cys (GRGDSPC) peptides and FITCGly-Arg-Gly-Asp-Ser-Pro-Cys (FITC-GRGDSPC) peptides were obtained from GL Biochem (Shanghai) Ltd. Water used in this study was distilled twice. This study was 
approved by the ethics committee of the Second Affiliated Hospital of Nanchang University, China.

\section{Synthesis and characterization of branched 4-arm-PEG-DA}

The branched PEG-DA used in this study was synthesized by us. The schematic representation of the synthesis is provided in Figure 1A. Briefly, linear PEG ( $M W=20 \mathrm{kDa}$ ) was dissolved in dichloromethane, and allowed to react with methanesulfonyl chloride, 5-hydroxyl-mono-methyl isophthalate, lithium aluminium tetrahydride, and acryloyl chloride, followed by purification procedures, to form branched PEG-DA. IR, ${ }^{1} \mathrm{H}$ NMR and ${ }^{13} \mathrm{C}$ NMR were used to verify the final product.

\section{PEGylation of decellularized valves}

The schematic representation for the PEGylation of decellularized valves (i.e. construction of PEG-DAV or PEG-crosslinking of decellularized valve) is presented in Figure 1B.

Porcine hearts were obtained from a local slaughterhouse. The porcine aortic valves were excised from the hearts under sterile conditions; decellularization was performed as described previously [18-20]. However, in this study, several modifications were applied. Briefly, after removal of adherent tissue the aortic valve leaflets were placed in a solution of $0.05 \%$ Triton-100 with $0.02 \%$ EDTA in phosphate buffer solution (PBS) without $\mathrm{Ca}^{2+}$ and $\mathrm{Mg}^{2+}$ for 24 hours under continuous shaking at $37^{\circ} \mathrm{C}$, together with RNase A $(0.02 \mathrm{mg} / \mathrm{mL})$ and DNase $(0.2 \mathrm{mg} / \mathrm{mL})$ followed by PBS supplemented with penicillin and streptomycin flushing for $48 \mathrm{~h}$ with constant shaking to remove all cellular debris. The valves were then stored in PBS at $4^{\circ} \mathrm{C}$ before further processing and seeding. To characterize the decellularized scaffolds, routine histological examination by hematoxylin and eosin (HE) and scanning electron microscopy (SEM) were performed [1,6,13,21].

SH-groups (Thiols) were introduced into the decellularized valve leaflets by a method similar to the one previously described [22]. Briefly, forty decellularized valve leaflets (mean dry weight $6.47 \pm 1.34 \mathrm{mg}$ per sample) were immersed in $50 \mathrm{~mL}$ of $50 \mathrm{mM}$ PBS (pH7.6, containing $1 \mathrm{mM}$ EDTA) and allowed to react with $50 \mathrm{mg}$ SATA for $2 \mathrm{~h}$ at room temperature under continuous shaking. The reaction was then stopped by extensive washing procedure with PBS. The acetylated sulfhydryl groups were deprotected by adding $50 \mathrm{~mL}$ of $0.5 \mathrm{M}$ hydroxylamine hydrochloride $\left(\mathrm{NH}_{2} \mathrm{OH} \cdot \mathrm{HCl}\right)$ in $50 \mathrm{mM}$ PBS (pH7.6) for $2 \mathrm{~h}$ at room temperature. Subsequently the leaflets were extensively washed with PBS to remove the redundant hydroxylamine hydrochloride. To test the extent of SH-modification, the leaflets which had been treated with SATA and hydroxylamine hydrochloride were allowed to react with $10 \mathrm{mM}$ DTNB (Ellman's reagent, 5,5'-dithiobis-[2-nitrobenzoicacid], Sigma) in $100 \mathrm{~mL}$ disodium hydrogen phosphate solution (50 mM, pH 8.0) for $15 \mathrm{~min}$. After determination of the absorbance at $412 \mathrm{~nm}$, the sulfhydryl group contents of the decellularized valve leaflets was deduced from standard curves obtained with cysteine [23]. Forty decellularized valve leaflets with introduced sulfhydryl groups were immersed in $50 \mathrm{~mL} 0.1 \mathrm{M} \mathrm{PBS}$ (pH7.5, $5 \mathrm{mM}$ EDTA), and allowed to react with $500 \mathrm{mg}$ branched 4-arm-PEG-DA for $8 \mathrm{~h}$ at $37^{\circ} \mathrm{C}$ under constant stirring. Finally, Samples were rinsed with PBS to remove the excess PEG-DA. Morphological analysis of PEGylated valves were performed using HE and SEM.

To evaluate the biomechanical properties of the PEGylated valves compared to native and decellularized valves, the leaflets specimens were subjected to uniaxial planar, tensile 


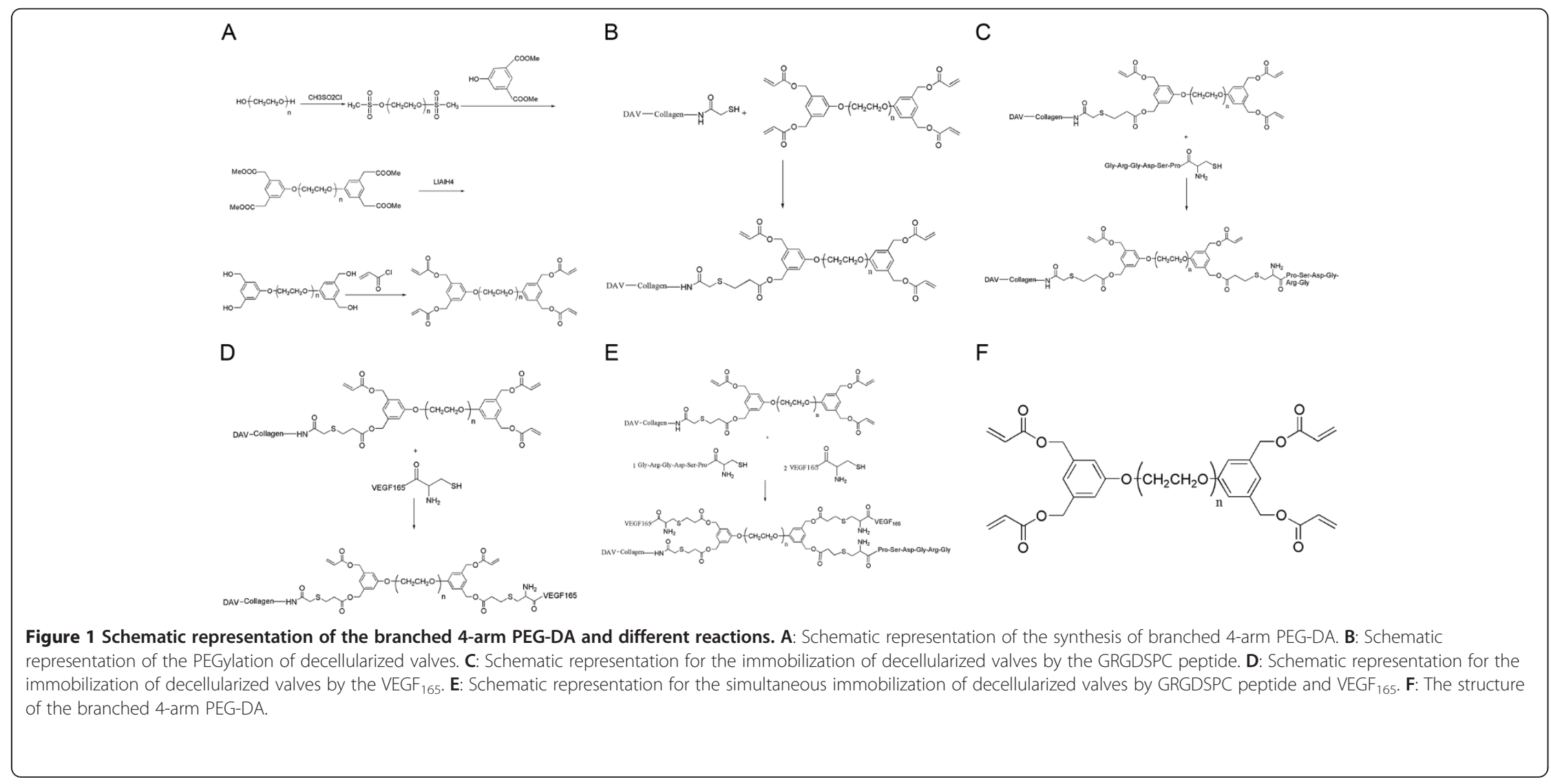


testing [13,24]. Specimens of $3 \mathrm{~mm} \times 15 \mathrm{~mm}$ were cut out from the valve leaflets in the circumferential fiber direction. The tensile tests were performed on an Instron 5848 MicroTester (Instron Corporation, U.S.A.) at a constant speed of $5 \mathrm{~mm} / \mathrm{min}$ until specimen fracture. Six specimens were tested in each group [6].

\section{Preparation of GRGDSPC-PEG-DAV-PEG-VEGF 165}

The GRGDSPC peptide and Human recombinant VEGF $_{165}$ was seperately immobilized covalently on decellularized valves by PEG-DA to find respective optimum reaction conditions (Schematic representations are provided in Figure 1(C,D)). The PEG-crosslinked decellularized valve was each reacted with $1 \mathrm{~mL}$ GRGDSPC peptide and Human recombinant $\mathrm{VEGF}_{165}$ solution of different concentrations at $37^{\circ} \mathrm{C}$. The amount of conjugation was evaluated quantitatively by spectrophotometry and ELISA at different time points, and the qualitative tests were performed by immunofluorescence (FITC-Ahx- Gly-Arg-Gly-AspSer-Pro-Cys, FITC-GRGDSPC,rabbit anti-human vascuoar endothelial cell growth factor and goat anti-rabbit IgG/Cy3). The decellularized valves were used as a negative control.

Considering respective optimum reaction conditions, The PEG-crosslinked decellularized valves were reacted with $1 \mathrm{~mL}$ reaction mixture solution (including $1 \mathrm{mg} / \mathrm{mL}$ GRGDSPC peptide and $1000 \mathrm{pg} / \mathrm{mL} \mathrm{VEGF}_{165}$ ) at $37^{\circ} \mathrm{C}$ for $4 \mathrm{~h}$. Then the immunofluorescence was analyzed for qualitative evaluation of the conjugation effects. The decellularized valves were used as a negative control (A schematic representation is provided in Figure 1E).

\section{Biological function of signal molecules immobilized covalently on decellularized valves}

After being cut into equal size disks (diameter $=1 \mathrm{~cm}$ ), DAVs, PEGylated decellularized aortic valves (PEG-DAV) and RGD and VEGF-conjugated PEGylated decellularized aortic valves (GRGDSPC-PEG-DAV-PEG-VEGF ${ }_{165}$ ) were put into different wells in a 24-well culture plate. HUVECs (KG110, Nanjing KeyGEN, China) [25] at 80\% confluence were harvested using a $0.05 \%$ trypsin solution and condensed to a concentration of $1 \times 10^{6} / \mathrm{mL}$ with serum-free media. A cell suspension of $0.5 \mathrm{~mL}$ was dropped on the top of the scaffolds and incubated in humidified air with $5 \% \mathrm{CO}_{2}$ at $37^{\circ} \mathrm{C}$ for $2 \mathrm{~h}$ to allow adherence of the cells onto the scaffolds. Following this incubation, unattached cells were removed with PBS, the cells/scaffolds constructs were then transferred into another 24-well plate and cultured for the desired time period.

The DNA content of the scaffold seeded HUVECs was quantified with Hoechst Dye 33258 (Invitrogen, USA) at 4 and 8 days. The cells were harvested from six samples of each group with $0.05 \%$ trypsin. Cells were centrifuged, lysed and then diluted lysates were incubated with an equal volume of $0.1 \mathrm{mg} / \mathrm{mL}$ Hoechst 33258 Dye for $5 \mathrm{~min}$ in 96-well plates protected from light. Fluorescence was determined with a FLUOstar Optima fluorescent plate reader (BMG Labtech, Offenburg, Germany) at $352 \mathrm{~nm}$ excitation and $461 \mathrm{~nm}$ emission. The DNA content in each sample was determined according to a DNA standard curve. SEM was performed for each group after 8 days of culture.

\section{Statistical analysis}

All quantitative data were expressed as mean \pm standard deviations (mean $\pm \mathrm{SD}$ ). Oneway analysis of variance (ANOVA) was performed and confidence intervals (CI) were determined using a statistical program (SPSS, Version 13.0, USA). $p$ values of less than 0.05 were considered statistically significant. 


\section{Results}

Identification of branched PEG-DA

The results of IR, ${ }^{1} \mathrm{H}$ NMR and ${ }^{13} \mathrm{C}$ NMR were as follows: IR (NaCl): $2883 \mathrm{~cm}^{-1}, 1723 \mathrm{~cm}^{-1}$, $1467 \mathrm{~cm}^{-1}, 1280 \mathrm{~cm}^{-1}, 1112 \mathrm{~cm}^{-1}, 946 \mathrm{~cm}^{-1}, 842 \mathrm{~cm}^{-1} ;{ }^{1} \mathrm{H}$ NMR $\left(\mathrm{CDCl}_{3}\right)$ : 3.51-3.86 $\left(\mathrm{OCH}_{2} \mathrm{CH}_{2}\right)_{\mathrm{n}}, 4.14\left(4 \mathrm{H}, \mathrm{CH}_{2} \mathrm{OAr}\right), 5.16\left(8 \mathrm{H}, \mathrm{CH}_{2}-\mathrm{Ar}\right), 5.88(4 \mathrm{H}, \mathrm{CH}=), 6.16\left(4 \mathrm{H}, \mathrm{CH}_{2}=\right)$, 6.44 (4H, $\left.\mathrm{CH}_{2}=\right)$, 6.90-6.96 (6H, Ar); ${ }^{13} \mathrm{C} \mathrm{NMR}\left(\mathrm{CDCl}_{3}\right): 166.2,159.3,137.5,131.2,128.0$, $113.9,70.5$. The structure of branched PEG-DA is given in Figure 1F.

Characteristics of decellularization and assessment of sulfhydryl group introduction into the decellularized valves

Decellularization procedures removed all cellular components of the valve leaflets, whereas the extracellular matrix was well preserved and showed a loosened and porous structure. The amount of sulfhydryl introduced into the decellularized valve (per mg dry weight) was $0.815 \pm 0.012 \times 10^{-7} \mathrm{~mol}$ by Ellman's reagent.

\section{Scanning electron microscopy}

Compared to decellularized valves, the surface of PEG-cross-linked decellularized valves suggested they could form a thin film of PEG, the connections between the collagenous fibers were significantly increased and fiber bundles were thickened (Figure 2).

\section{Mechanical testing of various valve leaflets}

It was found that the decellularization procedure reduced the maximal tensile strength of the acellular valves compared to the native valves $(\mathrm{P}<0.05)$. There was no significant difference with respect to the mechanical tensile strength properties between PEGylated and native groups, $\mathrm{n}=8$ (Figure 3 ).

\section{Characteristics of GRGDSPC-PEG-DAV-PEG-VEGF 165}

The GRGDSPC peptides were conjugated effectively with the PEG-cross-linked decellularized valves, shown by the FITC-GRGDSPC peptide giving high fluorescence in the PEG-crosslinked decellularized valves. However, the negative controls showed little fluorescence (Figure 4A). Moreover, the quantity of conjugated GRGDSPC peptides
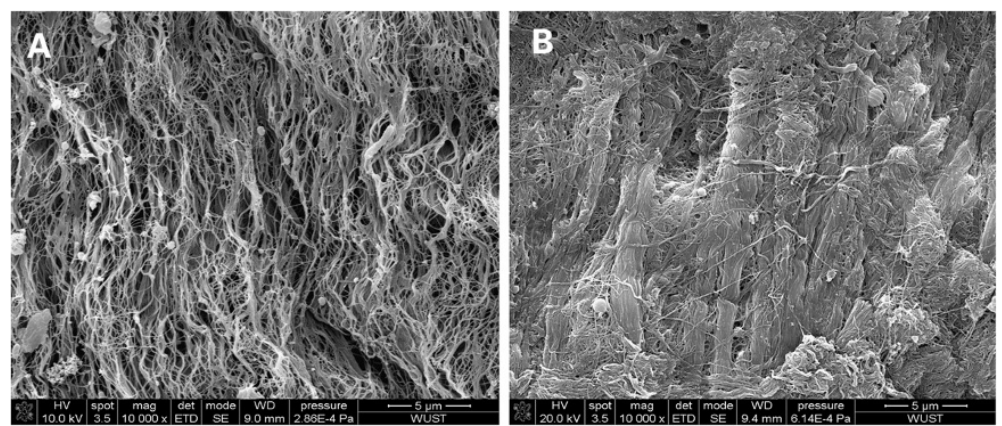

Figure 2 Observations of decellularized valve and PEG-cross-linked decellularized valve under scanning electron microscopy. SEM $(\times 10000)$ of a decellularized valve $(\mathbf{A})$ and a PEG-cross-linked decellularized valve (B). 


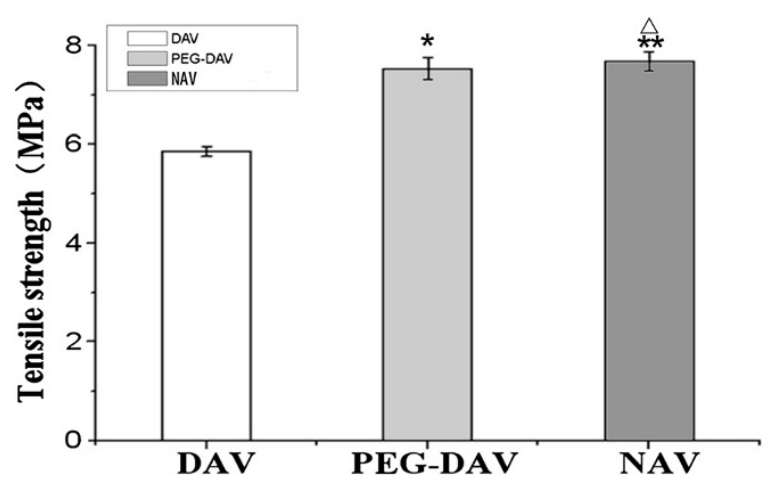

Figure $\mathbf{3}$ The maximal tensile strength as measured by using Instron 5848 MicroTester (Instron Corporation, U.S.A.). Decellularized aortic valves (DAV, $5.65 \pm 0.24$ ) vs. native aortic valves (NAV, 7.68 \pm 0.19 ): $p<0.05$; PEGylated decellularized aortic valves (PEG-DAV, $7.53 \pm 0.25$ ) vs. NAV: $p>0.05 n=8$

reached a maximum amount of $0.79 \pm 0.01 \mathrm{mg}$ when the concentration of GRGDSPC peptides in solution was $1 \mathrm{mg} / \mathrm{mL}$ and the reaction time was $2 \mathrm{~h}$ (Figure $4 \mathrm{~B}$ ).

The human recombinant VEGF $_{165}$ were conjugated effectively with the PEG-cross -linked decellularized valves, shown by the $\mathrm{VEGF}_{165}$ being highly positive in immunofluorescence in the PEG-cross-linked decellularized valves, but the negative controls showed little fluorescence (Figure 4C). Moreover, the quantity of conjugated VEGF 165 reached a maximum of $896.87 \pm 3.27 \mathrm{pg}$ when the concentration of $\mathrm{VEGF}_{165}$ in solution was $1000 \mathrm{pg} / \mathrm{mL}$ and the reaction time was $4 \mathrm{~h}$ (Figure 4D).

The GRGDSPC peptides and human recombinant VEGF $_{165}$ were conjugated effectively with the PEG-cross-linked decellularized valves simultaneously, since the GRGDSPC peptide and VEGF165 were highly positive in immunofluorescence of the PEG-cross-linked decellularized valves, but the negative controls showed little fluorescence (Figure 4E).

Biological function of the GRGDSPC peptide and human recombinant VEGF 165 immobilized covalently on decellularized valves: cellular immobilization and proliferation The DNA content of the GRGDSPC-PEG-DAV-PEG-VEGF 165 group at 4 and 8 days were $26.91 \pm 0.88 \mu \mathrm{g} / \mathrm{scaffold}$ and $30.59 \pm 1.42 \mu \mathrm{g} / \mathrm{scaffold}$, respectively, while those in the DAV were $24.24 \pm 0.68 \mu \mathrm{g} / \mathrm{scaffold}$ and $27.26 \pm 1.35 \mu \mathrm{g} / \mathrm{scaffold}$ and in the PEGDAV group were $23.28 \pm 1.25 \mu \mathrm{g} / \mathrm{scaffold}$ and $26.9 \pm 1.17 \mu \mathrm{g} / \mathrm{scaffold}$. The DNA content of the DAV and PEG-DAV groups were significantly lower than those of the GRGDSPCPEG-DAV-PEG-VEGF 165 group at 4 and 8 days (Figure 5A). Meanwhile, SEM revealed that a confluent and compact monolayer was formed on the surface of the GRGDSPCPEG-DAV-PEG-VEGF 165 group, but the other groups had clusters of scattered cells with endothelial morphology (Figure 5B).

\section{Discussion}

PEG is a hydrophilic polymer, which has a long flexible chain and is attractive to researchers into the polypeptide/protein drugs and molecular-targeted therapies because of its favorable biodegradability and biocompatibility [22,26,27]. Interestingly, functional groups [28] (such as vinyl sulphone, acryloyl) can be introduced into the terminal hydroxyl of PEG because of its strong controllability, which makes PEG widely used for chemical 


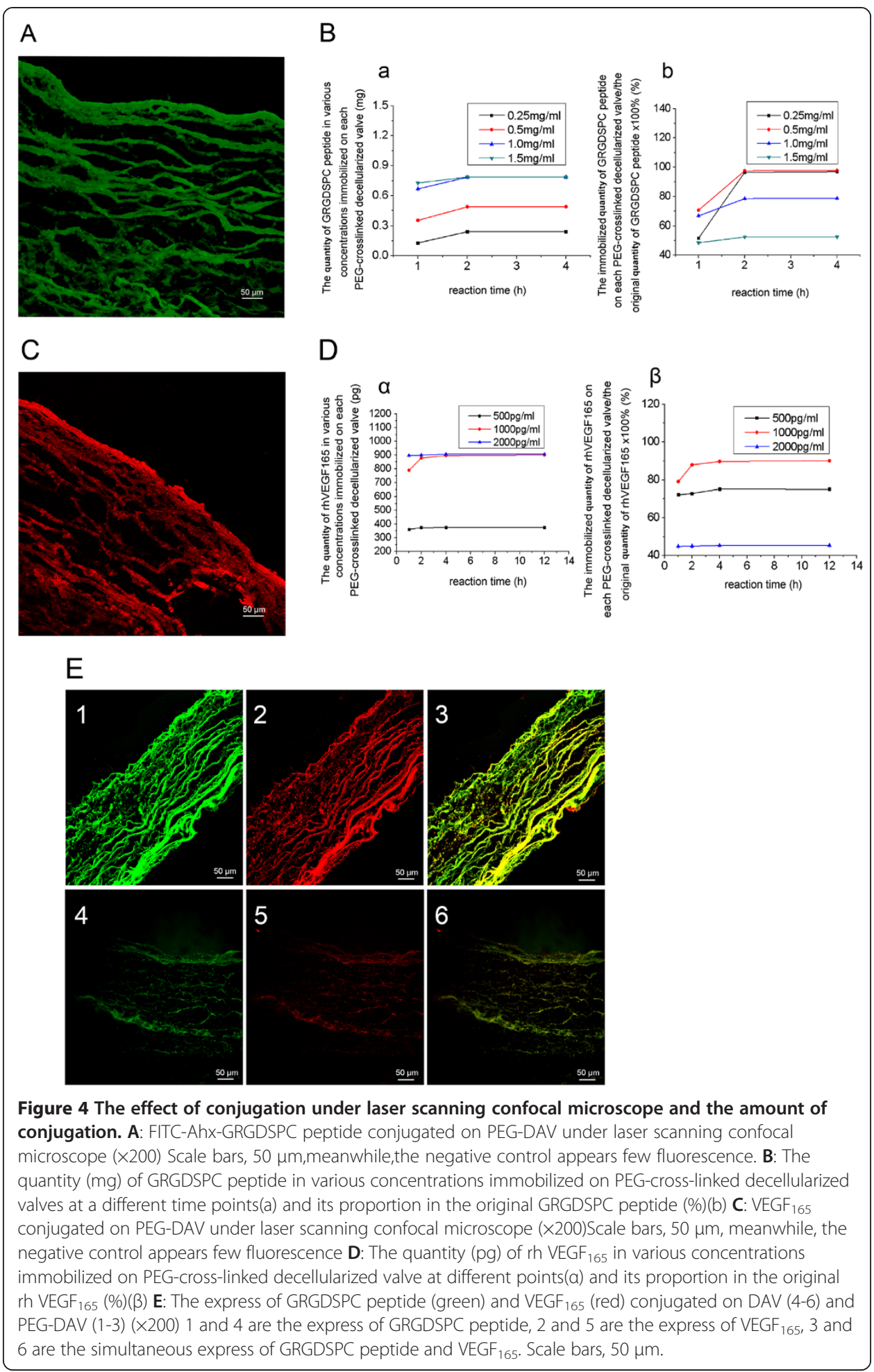

modification [29-34]. In the present study, the acroloyl-group was introduced into the end of every branch of PEG, which provided a method for the covalently binding of thiols.

PEGylation is the covalent attachment of PEG to biomolecules of interest, such as (poly) peptides and proteins. PEGylation was first reported by Abuchowski et.al in the 


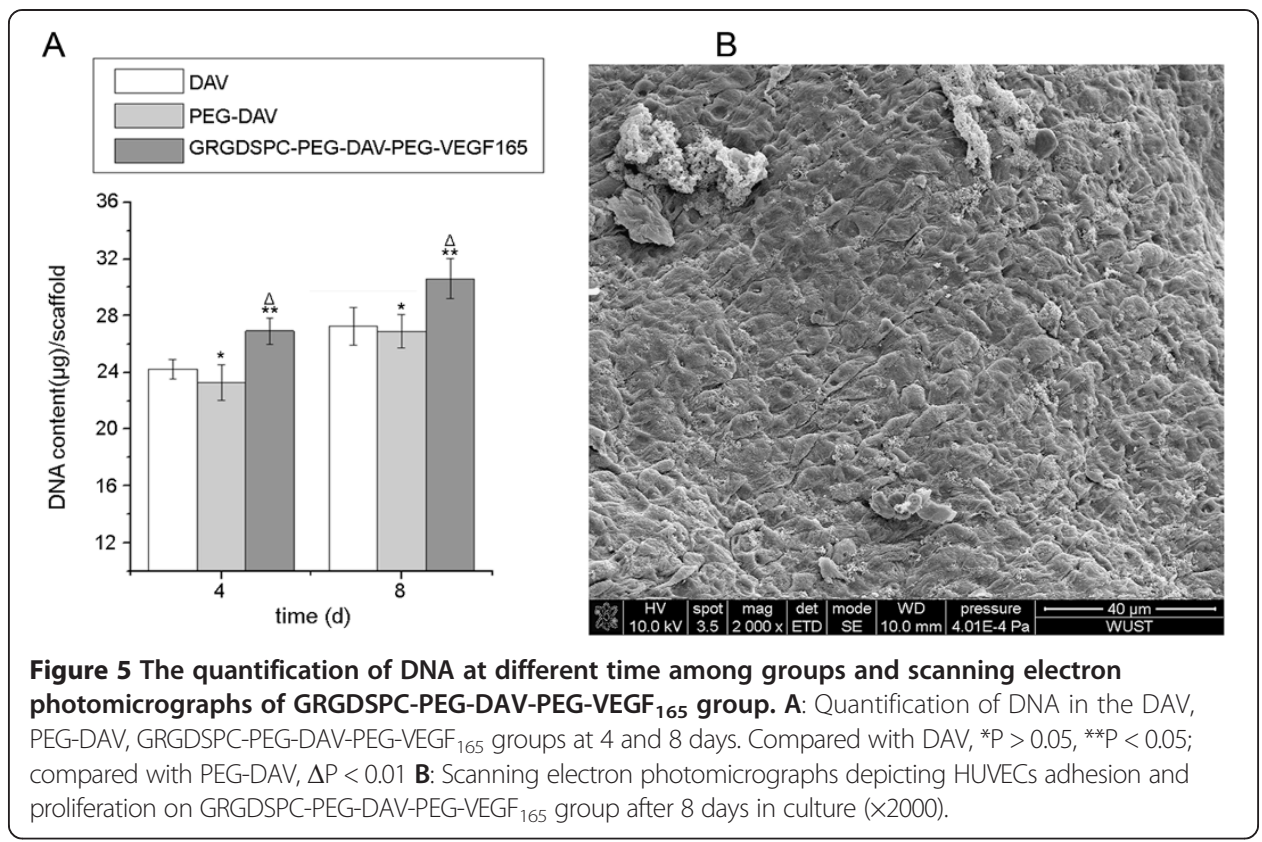

1970s for albumin and catalase modification [35,36]. Since then the procedure of PEGylation has been broadened and developed tremendously [37,38]. An important aspect of PEGylation is the incorporation of various PEG functional groups that are used to attach the PEG to the peptide or protein [39]. Collagen is one of the important components of the extracellular matrix of heart valves; decellularization retains most of the collagen component. Thus, PEGylation of decellularized valves is mainly PEGylation of collagen.

Crosslinking of decellularized valves required dual functionality of PEG-DA. For the first function, $\mathrm{SH}$-groups were introduced into the collagen by SATA, then with excess of acroloyl-groups on PEG-DA compared with the SH-groups on the decellularized valves (the molar ratio of acroloyl-groups to SH-groups was 20:1), PEG-crosslinking of decellularized valves was accomplished by the Michael addition reaction of the acroloyl-groups and the SH-groups. In this study, the SEM showed that the surface of the PEG-cross-linked decellularized valves formed a thin film of PEG, the connection between collagenous fibers increased significantly and formed fiber bundles that were thicker than with the decellularized valves. The biomechanical tests showed that the tensile strength of the PEG-cross-linked decellularized valves had been notably enhanced compared with decellularized valves and there was no obvious difference with native valves. The insertion of PEG molecules among the collagen molecules might have cross-linked the collagen molecules to one another by the addition reaction of acroloyl-groups and SH-groups, which in turn made the space structure of collagen in the decellularized valves more stable.

The second function of PEG-DA allows the immobilization of biological signals, such as RGD peptides and VEGF, immobilized covalently into decellularized valves is another function of bifunctional PEG-DA. Using the Michael addition reaction of unsaturated acroloyl-groups in PEG-cross-linked decellularized valves (i.e. unreacted acroloyl-groups in PEG with SH-groups introduced into decellularized valves) with $\mathrm{SH}$-groups in the 
GRGDSPC peptide or (and) VEGF, biological signal was immobilized covalently into decellularized valves. In this study, laser confocal microscopy observation and quantitative detection (spectrophotometry and ELISA) showed that GRGDSPC peptide and VEGF can not only be conjugated respectively, but can also be conjugated simultaneously by using PEG-DA. More importantly, these biological signals can not only be conjugated into decellularized valve, but also promote cell adhesion and cell proliferation, which was confirmed by the cellular DNA contents of each group and SEM.

\title{
Conclusions
}

Using the Michael addition reaction of acroloyl-groups in PEG-DA and SH-groups, not only the PEG-cross-linking of decellularized valves can be accomplished, and biological signals can also be conjugated into decellularized valves, which can partially improve the partial mechanical and biological properties of decellularized valves. Therefore, the novel branched PEG-DA may be a potential cross-linking reagent for the modification of tissue engineering scaffolds, which is expected to break through the traditional glutaraldehyde cross-linking of bio-derived valve materials, and to overcoming the shortcomings of valve decellularization. Further research and exploration of GRGDSPC-PEG-DAV-PEG-VEGF 165 should be made into their wear over time and fluid dynamic performance, as well as the interactions of signaling molecules cells and extracellular matrix in vivo or in a bioreactor.

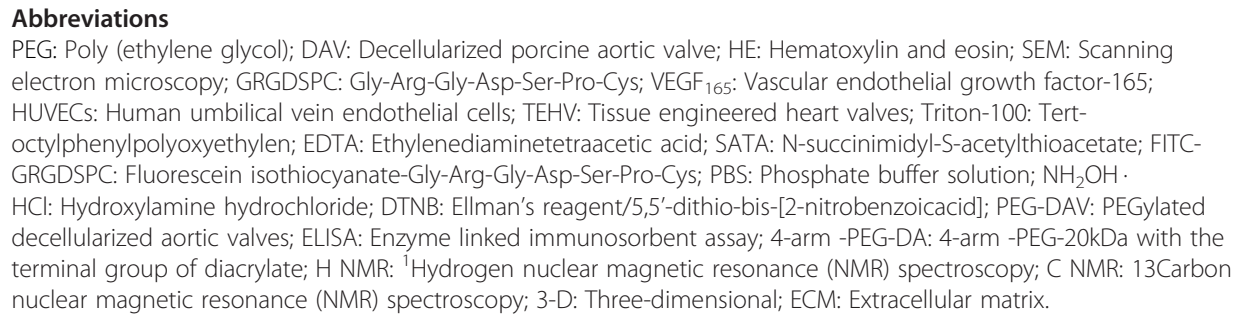

Competing interests

The authors declare that they have no competing interests.

\section{Authors' contributions}

Each Author has contributed substantially to the research, preparation and production of the paper and approves of its submission to the Journal. All authors read and approved the final manuscript.

Authors' information

Jianliang Zhou and Shidong Hu are co-first authors.

\section{Acknowledgements}

This work was supported by a grant from National Natural Science Foundation of China (NO. 81260047 and NO.81071271) and National High-tech Research and Development Program (863 Program) of China (No. 2009AA03Z420).

\author{
Author details \\ 'Department of Cardiothoracic Surgery, the Second Affiliated Hospital of Nanchang University, Nanchang 330006, \\ China. ${ }^{2}$ Medical College of Nanchang University, Nanchang 330006, China. ${ }^{3}$ Department of Cardiovascular Surgery, the \\ Union Hospital Affiliated to Tongji Medical College of Huazhong University of Science and Technology, Wuhan \\ 430022, China. \\ Received: 1 July 2013 Accepted: 4 September 2013 \\ Published: 5 September 2013 \\ References \\ 1. Dong X, Wei X, Yi W, Gu C, Kang X, Liu Y, Li Q, Yi D: RGD-modified acellular bovine pericardium as a \\ bioprosthetic scaffold for tissue engineering. J Mater Sci-Mater M 2009, 20:2327-2336. \\ 2. Filova E, Straka F, Mirejovský T, Mašín J, Bačáková L: Tissue-engineered heart valves. Physiol Res 2009, 58:S141-S158. \\ 3. Dohmen PM: Clinical results of implanted tissue engineered heart valves. HSR Proceedings In Intensive Care \& \\ Cardiovascular Anesthesia 2012, 4:225-231.
}


4. Dijkman PE, Driessen-Mol A, Frese L, Hoerstrup SP, Baaijens FP: Decellularized homologous tissue-engineered heart valves as off-the-shelf alternatives to xeno- and homografts. Biomaterials 2012, 33:4545-4554.

5. Simionescu DT, Chen J, Jaeggli M, Wang B, Liao J: Form follows function: advances in trilayered structure replication for aortic heart valve tissue engineering. J Healthcare Engineering 2012, 3:179-202.

6. Wu S, Liu YL, Cui B, Qu XH, Chen GQ: Study on decellularized porcine aortic valve/poly (3-hydroxybutyrate-co3-hydroxyhexanoate) hybrid heart valve in sheep model. Artif Organs 2007, 31:689-697.

7. Bader A, Schilling T, Teebken OE, Brandes G, Herden T, Steinhoff G, Haverich A: Tissue engineering of heart valveshuman endothelial cell seeding of detergent acellularized porcine valves. Eur J Cardio-Thorac 1998, 14:279-284

8. Booth C, Korossis SA, Wilcox HE, Watterson KG, Kearney JN, Fisher J, Ingham E: Tissue engineering of cardiac valve prostheses I: development and histological characterization of an acellular porcine scaffold. J Heart Valve Dis 2002, 11:457-462.

9. Schenke-Layland K, Vasilevski O, Opitz F, Konig K, Riemann I, Halbhuber KJ, Wahlers T, Stock UA: Impact of decellularization of xenogeneic tissue on extracellular matrix integrity for tissue engineering of heart valves. J Struct Biol 2003, 143:201-208.

10. Zhai W, Chang J, Lin K, Wang J, Zhao Q, Sun X: Crosslinking of decellularized porcine heart valve matrix by procyanidins. Biomaterials 2006, 27:3684-3690.

11. Ye XF, Hu X, Wang HZ, Liu J, Zhao Q: Polyelectrolyte multilayer film on decellularized porcine aortic valve can reduce the adhesion of blood cells without affecting the growth of human circulating progenitor cells. Acta Biomater 2012, 8:1057-1067.

12. Zhou J, Fritze O, Schleicher M, Wendel HP, Schenke-Layland K, Harasztosi C, Hu S, Stock UA: Impact of heart valve decellularization on 3-D ultrastructure, immunogenicity and thrombogenicity. Biomaterials 2010, 31:2549-2554.

13. Liao J, Joyce EM, Sacks MS: Effects of decellularization on the mechanical and structural properties of the porcine aortic valve leaflet. Biomaterials 2008, 29:1065-1074.

14. Mendoza-Novelo B, Avila EE, Cauich-Rodriguez JV, Jorge-Herrero E, Rojo FJ, Guinea GV, Mata-Mata JL: Decellularization of pericardial tissue and its impact on tensile viscoelasticity and glycosaminoglycan content. Acta biomater 2011, 7:1241-1248.

15. Tudorache I, Cebotari S, Sturz G, Kirsch L, Hurschler C, Hilfiker A, Haverich A, Lichtenberg A: Tissue engineering of heart valves: biomechanical and morphological properties of decellularized heart valves. J Heart Valve Dis 2007, 16:567-573. discussion 574 .

16. Somers $\mathrm{P}, \mathrm{De}$ Somer $\mathrm{F}$, Cornelissen $\mathrm{M}$, Thierens $\mathrm{H}$, Van Nooten G: Decellularization of heart valve matrices: search for the ideal balance. Artif Cells Blood Substit Immobil Biotechnol 2012, 40:151-162.

17. Vismara R, Soncini M, Talo G, Dainese L, Guarino A, Redaelli A, Fiore GB: A bioreactor with compliance monitoring for heart valve grafts. Ann Biomed Eng 2010, 38:100-108.

18. Steinhoff G, Stock U, Karim N, Mertsching H, Timke A, Meliss RR, Pethig K, Haverich A, Bader A: Tissue engineering of pulmonary heart valves on allogenic acellular matrix conduits: in vivo restoration of valve tissue. Circulation 2000, 102:III50-III55.

19. Grauss RW, Hazekamp MG, Oppenhuizen F, van Munsteren CJ, Gittenberger-de Groot AC, De Ruiter MC Histological evaluation of decellularised porcine aortic valves: matrix changes due to different decellularisation methods. Eur J Cardio-Thorac 2005, 27:566-571.

20. Shi J, Dong N, Sun Z: Immobilization of decellularized valve scaffolds with Arg-Gly-Asp-containing peptide to promote myofibroblast adhesion. J Huazhong Univ Sci 2009, 29:503-507.

21. Yang M, Chen CZ, Shu YS, Shi WP, Cheng SF, Gu YJ: Preseeding of human vascular cells in decellularized bovine pericardium scaffold for tissue-engineered heart valve: an in vitro and in vivo feasibility study. J Biomed Mater Res B 2012, 100:1654-1661.

22. Chen JS, Noah EM, Pallua N, Steffens GC: The use of bifunctional polyethyleneglycol derivatives for coupling of proteins to and cross-linking of collagen matrices. J Mater Sci-Mater M 2002, 13:1029-1035.

23. Riener CK, Kada G, Gruber HJ: Quick measurement of protein sulfhydryls with Ellman's reagent and with 4,4'-dithiodipyridine. Anal Bioanal Chem 2002, 373:266-276.

24. Christmann A, lenny P, Quantin J, Caro-Bretelle A, Lopez-Cuesta J: Mechanical behaviour at large strain of polycarbonate nanocomposites during uniaxial tensile test. Polymer 2011, 52:4033-4044.

25. Baudin B, Bruneel A, Bosselut N, Vaubourdolle M: A protocol for isolation and culture of human umbilical vein endothelial cells. Nat Protoc 2007, 2:481-485.

26. Jain N, Nahar M: PEGylated nanocarriers for systemic delivery. Methods Mol Biol 2010, 624:221-234.

27. Joralemon MJ, MCRae S, Emrick T: PEGylated polymers for medicine: from conjugation to self-assembled systems. Chem Commun (Camb) 2010, 46:1377-1393.

28. Ingavle GC, Dormer NH, Gehrke SH, Detamore MS: Using chondroitin sulfate to improve the viability and biosynthesis of chondrocytes encapsulated in interpenetrating network (IPN) hydrogels of agarose and poly (ethylene glycol) diacrylate. J Mater Sci-Mater M 2012, 23:157-170.

29. Davidovich-Pinhas $\mathrm{M}$, Bianco-Peled $\mathrm{H}$ : Novel mucoadhesive system based on sulfhydryl-acrylate interactions. J Mater Sci-Mater M 2010, 21:2027-2034.

30. Porter AM, Klinge CM, Gobin AS: Biomimetic hydrogels with VEGF induce angiogenic processes in both hUVEC and hMEC. Biomacromolecules 2011, 12:242-246.

31. Jevševar S, Kunstelj M, Porekar VG: PEGylation of therapeutic proteins. J Biotechnol 2010, 5:113-128.

32. Zhu J: Bioactive modification of poly(ethylene glycol) hydrogels for tissue engineering. Biomaterials 2010, 31:4639-4656.

33. Knop K, Hoogenboom R, Fischer D, Schubert US: Poly(ethylene glycol) in drug delivery: pros and cons as well as potential alternatives. Angew Chem Int Ed Engl 2010, 49:6288-6308.

34. Vanderhooft JL, Mann BK, Prestwich GD: Synthesis and characterization of novel thiol-reactive poly(ethylene glycol) cross-linkers for extracellular-matrix-mimetic biomaterials. Biomacromolecules 2007, 8:2883-2889.

35. Abuchowski A, van Es T, Palczuk NC, Davis FF: Alteration of immunological properties of bovine serum albumin by covalent attachment of polyethylene glycol. J Biol Chem 1977, 252:3578-3581. 
36. Abuchowski A, McCoy JR, Palczuk NC, van Es T, Davis FF: Effect of covalent attachment of polyethylene glycol on immunogenicity and circulating life of bovine liver catalase. J Biol Chem 1977, 252:3582-3586.

37. Dingels C, Wurm F, Wagner M, Klok HA, Frey H: Squaric acid mediated chemoselective pegylation of proteins: reactivity of single-step-activated alpha-amino poly(ethylene glycol)s. Chemistry 2012, 18:16828-16835.

38. Banerjee SS, Aher N, Patil R, Khandare J: Poly(ethylene glycol)-prodrug conjugates: concept, design, and applications. J Drug Del 2012, 2012:103973.

39. Roberts M, Bentley M, Harris J: Chemistry for peptide and protein PEGylation. Adv Drug Deliver Rev 2012, 64(supplement):116-127.

doi:10.1186/1475-925X-12-87

Cite this article as: Zhou et al:: Tissue engineering of heart valves: PEGylation of decellularized porcine aortic valve as a scaffold for in vitro recellularization. BioMedical Engineering OnLine 2013 12:87.

\section{Submit your next manuscript to BioMed Central and take full advantage of:}

- Convenient online submission

- Thorough peer review

- No space constraints or color figure charges

- Immediate publication on acceptance

- Inclusion in PubMed, CAS, Scopus and Google Scholar

- Research which is freely available for redistribution

Submit your manuscript at www.biomedcentral.com/submit

() Biomed Central 